\title{
Prospects for durable immune control of SARS-CoV-2 and prevention of reinfection
}

\section{Deborah Cromer, Jennifer A. Juno (D), David Khoury (1), Arnold Reynaldi(D, Adam K. Wheatley (D), Stephen J. Kent and Miles P. Davenport (1)}

Abstract | Immunity to severe acute respiratory syndrome coronavirus 2 (SARS-CoV-2) infection is central to long-term control of the current pandemic. Despite our rapidly advancing knowledge of immune memory to SARS-CoV-2, understanding how these responses translate into protection against reinfection at both the individual and population levels remains a major challenge. An ideal outcome following infection or after vaccination would be a highly protective and durable immunity that allows for the establishment of high levels of population immunity. However, current studies suggest a decay of neutralizing antibody responses in convalescent patients, and documented cases of SARS-CoV-2 reinfection are increasing. Understanding the dynamics of memory responses to SARS-CoV-2 and the mechanisms of immune control are crucial for the rational design and deployment of vaccines and for understanding the possible future trajectories of the pandemic. Here, we summarize our current understanding of immune responses to and immune control of SARS-CoV-2 and the implications for prevention of reinfection.

Severe acute respiratory syndrome coronavirus 2 (SARS-CoV-2) infection induces potent immune responses that have an important role in clearing a primary infection, including neutralizing antibodies and $\mathrm{CD}^{+}$and $\mathrm{CD}^{+} \mathrm{T}$ cell responses (reviewed in REF. ${ }^{1}$ ). Our understanding of immune memory to SARS-CoV-2 infection is progressing at an unprecedented pace, with studies of the duration of immune memory following closely behind the time course of the first known patients to be infected ${ }^{2-9}$. These analyses suggest, as would be expected from the normal contraction of the B cell response after other viral infections, that neutralizing antibody responses to SARS-CoV-2 peak within the first few weeks after symptom onset but seem to decay after this time, with a proportion of individuals losing detectable neutralizing antibodies within months after infection ${ }^{5}$. Cellular responses to infection seem to be generally more stable ${ }^{3,8,10}$ (FIG. 1) but their role in immune protection is not yet clear (as discussed in more detail below).
However, although our understanding of the magnitude and phenotype of immune responses to SARS-CoV-2 is progressing rapidly, discerning what is necessary and/or sufficient for protection remains a major challenge. That is, different immune responses may contribute singly or in combination to blocking initial infection, controlling viral replication, limiting pathogenesis and/or reducing onward transmission.

Despite some studies suggesting the persistence of detectable immune responses to SARS-CoV-2 $\left(\mathrm{REFS}^{8,10}\right)$, documented examples of SARS-CoV-2 reinfection as well as evidence of short-lived immunity to endemic human coronavirus (hCoV) infections raise the prospect that protective immunity after SARS-CoV-2 infection may last for months instead of years. Indeed, epidemiological studies suggest that, even in communities with high infection rates, herd immunity through natural infection may not have been achieved, and these studies raise the possibility that a combination of waning immunity and viral antigenic variation may allow for continued circulation of the virus ${ }^{11,12}$. In this context, it will be crucial to understand how natural and vaccine-induced immunity might shape the future transmission and pathogenicity of SARS-CoV-2.

Here, we argue that a better understanding of how immune memory translates to clinical protection at the patient and population levels is crucial to predicting the future of the pandemic. We discuss our understanding of immunity and immune memory following SARS-CoV-2 infection. We then ask how these responses can function together to provide resistance to reinfection and/or control viral replication during subsequent infections. Finally, we discuss what lessons can be learnt from our understanding of immunity to endemic $\mathrm{hCoV}$ and influenza virus infections and how these lessons can be applied to SARS-CoV-2.

\section{Immune responses to SARS-CoV-2} Neutralizing antibodies. Neutralizing antibodies are able to bind to the virus and directly block its ability to infect cells (usually through inhibition of the interaction between the viral spike protein and the cellular ACE2 receptor). Neutralizing antibodies are thought to have a crucial role in limiting viral replication within the host as well as the potential to neutralize virus at the point of entry before infection of host cells can occur. Studies suggest that serological neutralizing antibody responses peak within 3-5 weeks after infection, with the magnitude of the peak being correlated with the severity of clinical illness ${ }^{13}$. However, neutralizing antibody responses decay rapidly in early convalescence ${ }^{9,14-17}$. Estimates of the halflife of the neutralization titre over the first 70 days after infection suggest an early half-life of $\sim 55$ days $^{3}$, whereas a longer-term analysis suggested an overall half-life of 90 days over the first 8 months after infection ${ }^{8}$. Analysis of results from these and other studies is summarized in FIG. 1 a, generally showing similar patterns of decay (includes preprint data, not yet peer reviewed $)^{2,5,7,8,14-20}$. This pattern of rapid early decay is due to the short half-life of serum antibodies and to short-lived 


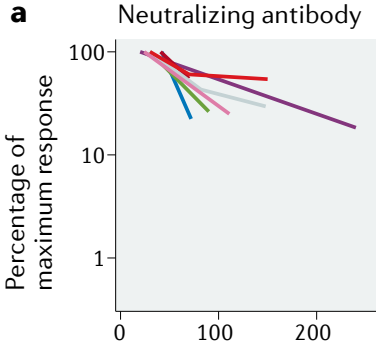

$\lg G$

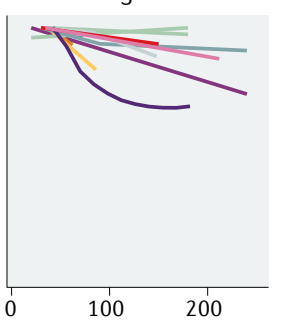

$\lg A$

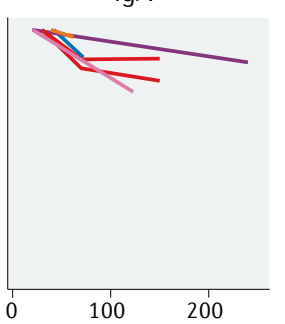

$\mathrm{CD}^{+} \mathrm{T}$ cells

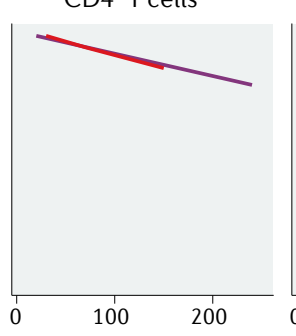

CD8 ${ }^{+} \mathrm{T}$ cells

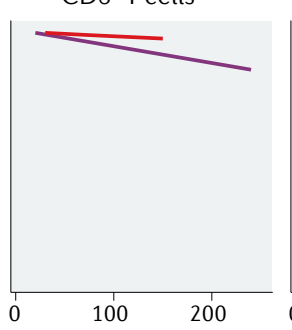

Memory B cells

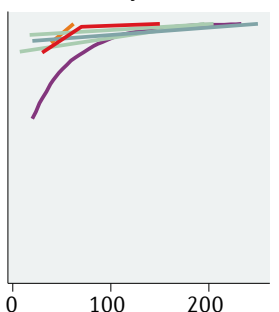
References
— Wheatley et al. $(2021)^{3}$
— Dan et al. (2021)
- Muecksch et al. $(2021)^{5}$
— Wajnberg et al. (2020) ${ }^{7}$
— Beaudoin-Bussières et al. (2020) ${ }^{17}$
— Gaebler at al. (2021)
_ Sokal et al. (2021) ${ }^{19}$
— Wu et al. $(2021)^{16}$
— Crawford et al. (2020) ${ }^{15}$
_ Ibarrondo et al. (2020) ${ }^{14}$
— Ellebedy et al. $(2020)^{20}$ (preprint) — Yao et al. (2020) ${ }^{18}$ (preprint)

b

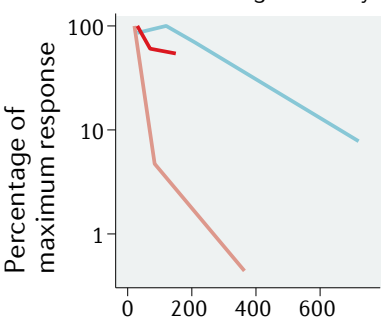

$\lg G$

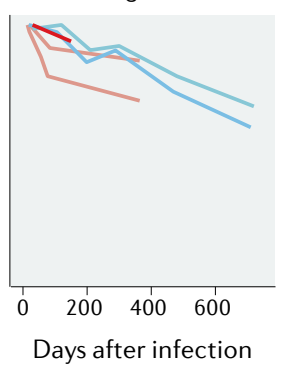

$\lg \mathrm{A}$

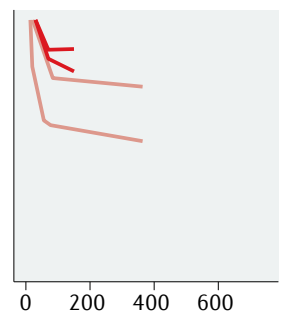

C

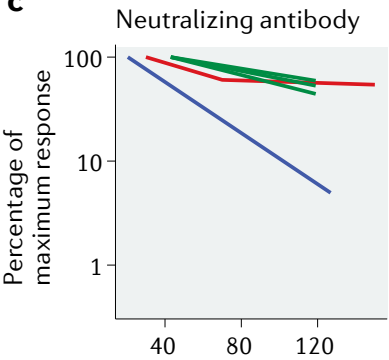

Total immunoglobulin

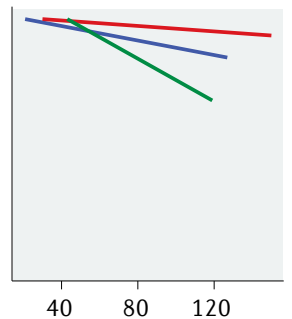

Days after infection or vaccination

$$
\begin{aligned}
& \text { References } \\
& \begin{array}{ll}
\text { - SARS-CoV-2 (Wheatley et al. } \left.(2021)^{3}\right) & \text { - SARS-CoV (Tang et al. } \left.(2011)^{90}\right) \\
\text { - SARS-CoV (Liu et al. } \left.(2006)^{89}\right) & \left.-\mathrm{hCoV} \text { (Callow et al. }(1990)^{76}\right)
\end{array}
\end{aligned}
$$

Fig. 1 | The decay of immune memory to coronavirus infections. a|Dynamics of immune decay following severe acute respiratory syndrome coronavirus 2 (SARS-CoV-2) infection. The rates of loss of SARS-CoV-2 neutralizing antibodies and binding antibodies ( $\lg G$ and $\lg A)$ as well as of $\mathrm{CD} 4^{+} \mathrm{T}$ cells, $\mathrm{CD} 8^{+} \mathrm{T}$ cells and memory $\mathrm{B}$ cells are shown as documented by several independent studies (includes preprint data, not yet peer reviewed) $)^{2,5,7,8,14-20,69,76,89-91}$. Neutralizing antibody responses show a biphasic decay over the first months after infection ${ }^{3}$. The rapid decay observed in the neutralization titre in the first $1-2$ months after infection is consistent with the decay kinetics of $\lg A$ responses and $\lg \mathrm{M}$ responses (not shown). The slower decay of neutralization titre after 2 months is more consistent with the slow decay of $\mathrm{lg} G$ responses ${ }^{3}$. T cell responses decay at a slightly slower rate than $\mathrm{lgG}$ over the first few months after infection, whereas memory $\mathrm{B}$ cell responses increase. The plotted data are derived from the half-lives reported directly by the authors of each study or from our calculation of halflives based on raw data extracted from the original publication. Details of the original studies, data extraction and analysis are provided in Supplementary Table 1. Direct comparisons of the absolute magnitude of responses between studies are not possible owing to the different assays used. However, the rate of decay for each study as a percentage of the maximum uses a consistent measure over time. $\mathbf{b}$ | Comparison of the kinetics of antibody decay following infection with SARS-CoV 89,90 or human coronavirus $(\mathrm{hCoV})^{76}$ and following mild-to-moderate SARS-CoV-2 infection ${ }^{3}$. A high variability is seen between studies; however, a rapid early decay followed by a slowing decay is seen for most serological responses. $\mathbf{c}$ /Comparing the durability of vaccine-induced ${ }^{69,91}$ and natural ${ }^{3}$ immunity to SARS-CoV-2. There is no evidence that vaccine-induced responses are more durable than convalescent responses (preprint data, not yet peer reviewed) ${ }^{24}$. antibody-secreting cells, which decay with a half-life of weeks to leave a population of long-lived plasma cells with half-lives of months to years ${ }^{21}$. It is likely that longerterm follow-up studies will reveal a further slowing of decay, with titres reaching a stable level analogous to humoral responses to other viral pathogens ${ }^{22,23}$.

Analysis of the relationship between neutralizing antibody titre and protection using data from vaccination and convalescent studies suggests that a neutralizing titre equivalent to $20 \%$ of the average convalescent titre is sufficient to provide $50 \%$ protection from symptomatic coronavirus disease 2019 (COVID-19; preprint data, not yet peer reviewed ${ }^{24}$. Combining data on the protective titre and dynamics of decay of neutralizing antibodies suggest that neutralization-mediated protection may decline substantially over the first year of infection and will also be significantly affected by the reduced neutralization of viral antigenic variants ${ }^{8,24}$. However, even if the level of neutralizing antibodies is insufficient to block viral entry and early replication, it is possible that lower levels of neutralizing antibodies can still act to slow viral growth rates and reduce the severity of infection (FIC. 2). Consistent with this, a lower neutralization titre (3\% of the average convalescent antibody titre) was associated with $50 \%$ protection from severe COVID-19 (REF. ${ }^{24}$ ). Understanding the role of neutralizing antibodies in the protection from SARS-CoV-2 infection and the clinical implications of antibody decay and antigenic variation remain major areas for future investigation. 
Non-neutralizing antibodies. Antibodies that bind to viral proteins but do not neutralize SARS-CoV-2 could still contribute to the immune control of infection through the increased clearance of free virus or by targeting infected cells for immune clearance (through antibody-dependent cellular cytotoxicity and other mechanisms $)^{25}$ (preprint data, not yet peer reviewed) ${ }^{26}$. Titres of SARS-CoV-2-binding antibodies can have markedly different decay kinetics to serological neutralizing activity, with differences also between antibody isotypes. Spike-specific IgG antibodies have an estimated half-life of 100-230 days ${ }^{3,8}$. By contrast, IgM and IgA1 spike-binding antibodies have shorter half-lives of 55 days and 42 days, respectively, early after infection (before day 70 post-infection), which is similar to the early decay rate of the neutralizing antibody titre; the decline of $\operatorname{IgM}$ and $\operatorname{IgA} 1$ responses then slows to a half-life of 118 days and $>1,000$ days, respectively, beyond 70 days after infection ${ }^{3}$. Another study estimated an average halflife of IgA antibodies of 210 days over the first 8 months after SARS-CoV-2 infection ${ }^{8}$. Thus, residual SARS-CoV-2-specific nonneutralizing antibodies following infection may confer some protective benefit during reinfection, even when serum neutralizing activity has declined below the threshold of protection $^{25-27}$.

Memory B cells. Memory B cells provide an additional mechanism for the durable maintenance of humoral immunity by providing for the rapid recall and production of protective antibodies following pathogen re-encounter. We and others have shown that SARS-CoV-2-specific memory B cells accumulate over the first few months after initial infection ${ }^{2,3,8,9,28}$ (FIG. 1a); increasing levels of antibody somatic mutations suggest that ongoing germinal centre activity drives the further affinity maturation of antibody responses over time $e^{2}$. The stable maintenance of a reservoir of affinity-matured memory $B$ cells could provide a mechanism to help mitigate subsequent infections, although the kinetics of recall and protective potential of memory B cells in SARS-CoV-2 immunity are yet to be determined.

\section{Memory $\mathrm{CD}^{+}$and $\mathrm{CDB}^{+} \mathrm{T}$ cells.}

Longitudinal studies have also characterized the $\mathrm{T}$ cell response to spike protein or other SARS-CoV-2 antigens ${ }^{3,8,29,30}$. Two studies observed similar half-lives for spike-specific $\mathrm{CD} 4^{+}$memory $\mathrm{T}$ cell

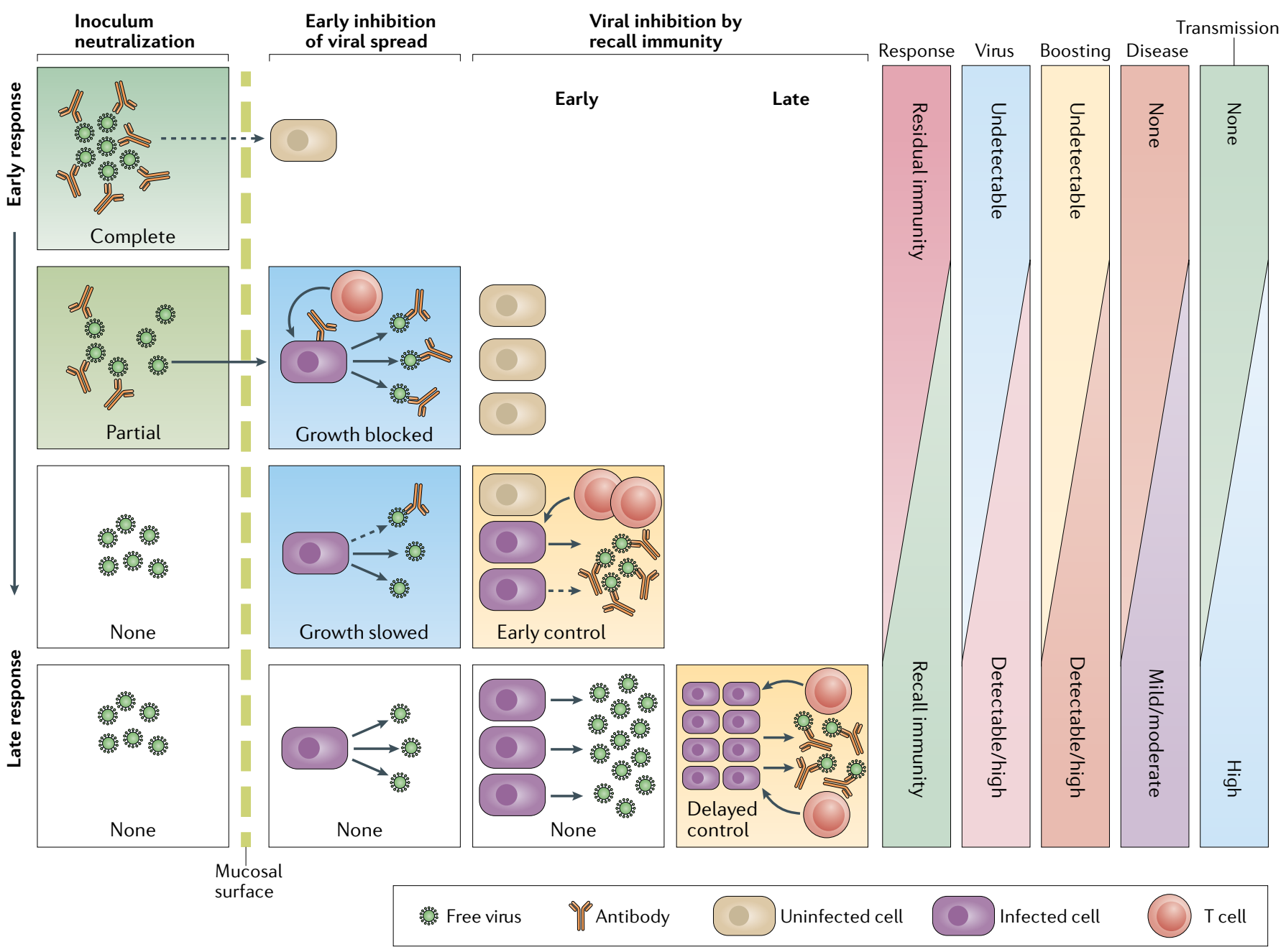

Fig. 2 | Immune control of SARS-CoV-2 reinfection. Immune memory could function to control severe acute respiratory syndrome coronavirus 2 (SARS-CoV-2) infection at several stages. Residual levels of neutralizing antibodies may block the entry of virus into host cells or prevent the early dissemination of virus. Even if viral growth is established, residual antibody and cellular responses may function to slow viral growth, providing a longer window of time for the action of recall immune responses. Recall responses start to take effect later in infection, boosting immune-mediated control and reducing the virus to low levels. Depending on the timing and efficacy of immune control, infection may vary from undetectable or mild, through to severe infection with viral detection and immune boosting. 
responses (120-139 days) and reported a high (>90\%) frequency of convalescent individuals having $\mathrm{CD} 4^{+} \mathrm{T}$ cell responses for 6 months after symptom onset ${ }^{3,8}$ (FIG. 1a). $\mathrm{CD}^{+} \mathrm{T}$ cell responses are also induced following SARS-CoV-2 infection, and they seem to have a longer half-life relative to the $\mathrm{CD}^{+} \mathrm{T}$ cell response $\mathrm{e}^{3,8}$. Although the impact of T cell-mediated immunity on COVID-19 pathogenesis is not well understood, there is some evidence linking robust $\mathrm{T}$ cell responses to mild disease outcome $^{31}$. In addition to the direct antiviral effector functions of T cells, $\mathrm{CD} 4^{+} \mathrm{T}$ follicular helper $\left(\mathrm{T}_{\mathrm{FH}}\right)$ cells promote antibody responses by supporting the development of germinal centre B cells. SARS-CoV-2 spike-specific circulating $\mathrm{T}_{\mathrm{FH}}$ cell responses correlate with the generation of neutralizing antibodies in convalescent individuals, particularly when these cells have a CCR6phenotype $^{32}$. The presence and phenotype of $\mathrm{T}_{\mathrm{FH}}$ cells in lymph nodes that drain the sites of infection, and the factors associated with generating $\mathrm{T}_{\mathrm{FH}}$ cell responses are not well understood at present but are likely to be crucial in maintaining robust antibody-based immunity. Consequently, a diverse array of $\mathrm{T}$ cell responses directed towards SARS-CoV-2 antigens are likely present in convalescent individuals at sufficient levels to mount a recall response upon reinfection. The role of $\mathrm{T}$ cell responses upon re-exposure to SARS-CoV-2 in humans is not known but, by analogy with other viral infections, they may reduce viral levels and severe disease upon re-exposure by limiting viral replication to the upper respiratory tract ${ }^{8,33}$.

\section{From immune responses to control}

It remains unclear which responses for example, humoral versus cellular responses - are necessary and/or sufficient to provide protective immunity against SARS-CoV-2 reinfection. In addition, both the maintenance and role of tissue-resident memory B cells and T cells in the lungs are not well understood as most studies so far have focused on responses in blood. The presence of memory cells at the site of infection may be crucial for efficient target recognition and immune recall ${ }^{34,35}$. It also seems likely that vaccination and natural infection may lead to different responses in the lung and at mucosal sites, further emphasizing the need to understand local responses.

Importantly, protective immunity itself is not a single outcome but might be considered as a spectrum involving the interplay of viral replication, immune control and pathogenesis (FIG. 2). At one extreme, high-level protective immunity may result in the prevention or early elimination of infection (before an immunological or pathological effect is detectable in the host). In the absence of such strong immunity, varying levels of viral replication and immune recall are possible, leading to different levels of clinical illness and the potential for transmission (BOX 1). Thus, the decay of immune memory over time is unlikely to produce a switch from an 'immune' to a 'non-immune' status but rather a gradual reduction in immunity and perhaps even a transfer between different immune responses that have a leading role in protection at different times.

The varying mechanisms of action and dynamics of different immune responses suggest that they may be involved at different stages following reinfection (FIG. 2). Neutralizing antibodies, for example, may function immediately to block the initial infection of host cells through residual levels of IgA or IgG at the mucosal surface. If a virus succeeds in passing this barrier, circulating IgG may prevent the establishment of infection by neutralizing the initial inoculum or by blocking subsequent viral spread. Even if neutralizing IgG cannot fully block infection, it may still slow the rate of growth of SARS-CoV-2, creating a larger time window for memory responses to be recalled and expand to contribute to controlling infection before the viral peak (BOX 1).

It is important here to differentiate between the roles and effects of 'residual' and 'recall' immune responses to infection. Antibodies, for example, can be maintained in the circulation or within tissues for prolonged periods after an initial period of rapid decay (as discussed above), and this residual level of antibodies can function immediately to neutralize incoming virus. Similarly, persistent tissue-resident effector cells may be poised for early action at the site of infection. However, in addition to these residual responses that are present before infection occurs, immune responses can be boosted by the recall of memory $B$ cells and $\mathrm{T}$ cells, which can be activated and proliferate to rapidly supplement humoral and cellular immunity soon after infection. These recalled immune responses may, if sufficiently rapid, function to control peak viral levels and reduce disease severity. Evidence for this comes from studies involving the administration of SARS-CoV-2-specific monoclonal antibodies within the first week of symptoms in mild-to-moderate SARS-CoV-2 infection, which seems to accelerate the clearance of virus and reduce the risk of clinical progression ${ }^{36,37}$. This effect was dose dependent and was greater for those patients who lacked endogenous antibody responses. Studies of passive transfer of immune serum to patients later in the disease course with severe COVID-19 have had mixed outcomes, with some studies suggesting that boosting antibody levels at a later time point might improve clinical outcomes ${ }^{38,39}$ but other studies showing no effect ${ }^{40}$. Early results suggest that monoclonal antibody cocktails may be useful in prophylaxis or early disease but are less effective in treating patients who are critically ill when inflammation dominates the clinical picture ${ }^{41}$.

Taken together, these results strongly support the idea that early boosting of antibody levels by recall immunity could have an important role in limiting the severity of clinical disease. Thus, in addition to measuring circulating antibody levels, identifying the durability, specificity and recall kinetics of B cell memory may be crucial for understanding and predicting the durability of protection from SARS-CoV-2 infection. The observations that frequencies of circulating memory B cells continue to increase for several months after recovery from COVID-19 ( REFS $^{2,3,8,9,28}$ ), when most other responses are declining (FIC. 1 a), suggest that recall of B cell memory may provide a mechanism to extend the duration of antibody-dependent protection in the face of waning serological titres.

The role of cellular immunity in protection from SARS-CoV-2 similarly depends on the kinetics of the response and capacity to control viral replication. Studies of the ability of memory T cells to control viral and bacterial growth suggest that both $\mathrm{CD} 4^{+} \mathrm{T}$ cells (in murine tuberculosis ${ }^{42}$ ) and $\mathrm{CD}^{+} \mathrm{T}$ cells (in simian immunodeficiency virus infection of macaques $\left.{ }^{43,44}\right)$ require an extended period of infection (7-10 days) before they are mobilized to control pathogen replication. It is not clear whether similar dynamics underpin the recall of SARS-CoV-2-specific $\mathrm{T}$ cell memory as they may be quite pathogen specific $^{45}$, and further studies are warranted to define the protective contribution of memory T cells in SARS-CoV-2 immunity.

\section{Correlates of immune protection}

The contribution of different aspects of immune memory to the protection against SARS-CoV-2 reinfection in humans remains unclear. Recent analyses suggest that neutralizing antibodies are a strong correlate of vaccine-induced immunity in humans (preprint data, 


\section{Box 1 | Beyond sterilizing immunity?}

Severe acute respiratory syndrome coronavirus 2 (SARS-CoV-2) infection leads to a range of clinical as well as virological outcomes in different nonimmune individuals (see figure, part a). The colour gradient from green to red indicates the correlation between viral levels and severity of illness. The relationship between viral levels and transmission may not be linear (three potential example relationships are indicated).

A classical view of vaccination is that it provides 'sterilizing immunity', in which vaccinated individuals experience such strong immune protection that they do not become infected when later exposed. That is, we could think of vaccine-mediated protection as an 'all or nothing' phenomenon, where vaccine efficacy reflects the proportion of individuals who are completely protected from infection (and all other individuals have an unchanged risk of infection) (see figure, part b). In this paradigm, the 'herd immunity threshold' for vaccination ${ }^{87,88}$ is the proportion of individuals who need to be 'fully protected' in order to reduce transmission of the pathogen below the level of replacement (in other words, so that each infected individual infects less than one other individual and the effective reproductive number drops below 1 ).

However, this binary picture of immune protection is likely not the normal outcome of most infections or vaccinations. Instead, individuals with different levels of immunity will suppress the virus to a variable extent (which will also change within the same individual over time as immunity wanes) ${ }^{24}$, and immunity functions to reduce average viral levels across the population. This more complex landscape of viral control could markedly impact the burden of disease within a population by reducing viral replication, the severity of illness and/or transmission (see figure, part c). A proportion of individuals may still experience mild infection and a proportion may be fully protected.

If immunity is insufficient to block viral replication, then any ongoing transmission is expected to reboost immune responses, leading to a long-term equilibrium of transmission and immune boosting ${ }^{82}$. Finally, it is not clear that clinical protection and transmission should necessarily be reduced by the same amount. Immunity need not decrease viral levels evenly across the spectrum of potential responses. For example, if recall immune responses function to prevent high peak viral levels (but still allow for lower viral levels) then, depending on the relationship
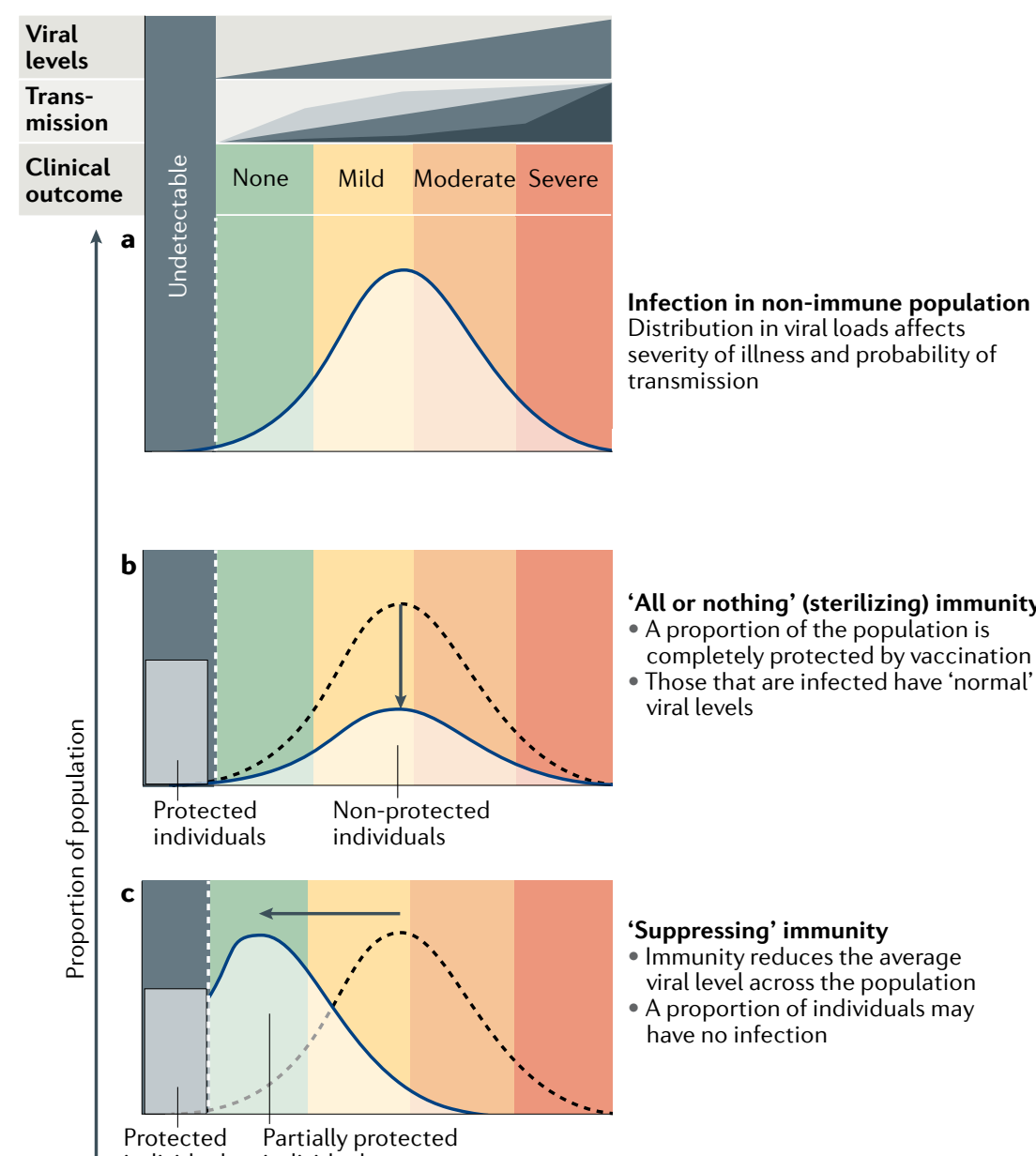

'All or nothing' (sterilizing) immunity - A proportion of the population is completely protected by vaccination - Those that are infected have "normal' viral levels

'Suppressing' immunity

- Immunity reduces the average viral level across the population - A proportion of individuals may have no infection

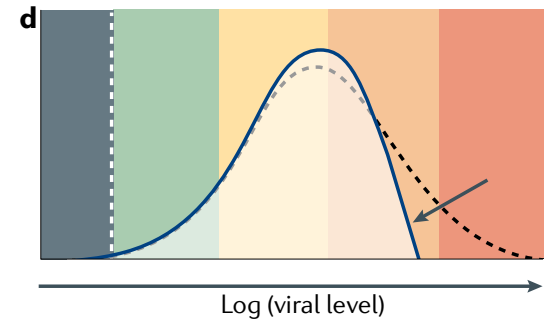

'Peak-reducing' immunity Immunity is unable to control moderate infection but prevents high peak viral loads from causing severe illness

between viral levels and transmission, there may be a greater or lesser reduction in transmission for a given level of clinically observed protection (see figure, part d).

not yet peer reviewed $)^{24}$. Preliminary studies in animal models have also been informative. Initial studies in macaques showed that a primary infection with SARS-CoV-2 protects against subsequent viral challenge for at least 28-35 days, with re-exposure driving memory immune responses that included boosting of binding and neutralizing antibody levels as well as of IFN $\gamma$ secretion by $\mathrm{T}$ cells ${ }^{46,47}$. Further studies have provided evidence for a contribution of both IgG antibody and $\mathrm{CD}^{+} \mathrm{T}$ cells in providing protection against reinfection ${ }^{48}$. The transfer of high titres of purified IgG from convalescent animals is sufficient to protect against viral challenge or, at lower titres, to reduce the duration of viral replication. In addition, the depletion of $\mathrm{CD}^{+} \mathrm{T}$ cells at 7 weeks after infection in macaques resulted in a partial loss of protection from re-challenge $e^{48}$. These data suggest that, in the context of declining neutralizing antibody titres, cellular immunity is required to provide maximal protective immunity against SARS-CoV-2 re-exposure ${ }^{48}$. However, despite these mechanistic insights provided by animal studies, it is often impractical to assess long-term immunity ( $>6$ months after infection). Nevertheless, although animal models may not recapitulate all of the features of human infection ${ }^{49}$, they suggest the importance of looking beyond neutralizing antibodies for additional mechanisms of immune protection.

\section{SARS-CoV-2 reinfection in humans}

Despite the robust, multi-faceted immune memory generated by SARS-CoV-2 infection that we describe above, there have 
been a small number of well-documented cases of reinfection with SARS-CoV-2 (Supplementary Table 2). However, there is likely a significant under-reporting of SARS-CoV-2 reinfections owing to, among other things, a lack of resources with which to sequence all infections and a tendency to attribute a subsequent infection to 'long COVID'. In addition, individuals with low levels of infection who do not have clinically significant symptoms may not present for screening, which suggests that current evidence of reinfections may be the 'tip of the iceberg' of recurrent infection events. Once a potential reinfection case is identified, conclusive determination can be confounded by recurrent symptoms following an initial infection, persistent shedding of SARS-CoV-2 RNA, the potential for laboratory false positives, minor evolution of SARS-CoV-2 variants within a host, and the variability and timing of antibody responses to the initial infection. Nonetheless, reinfections with clearly different SARS-CoV-2 strains have now been documented ${ }^{50,51}$ (Supplementary Table 2). A comprehensive analysis of more than 133,000 cases documented 54 cases that were considered to have strong evidence for reinfection with SARS-CoV-2 at least 45 days after the initial infection, of which 4 cases were confirmed to have a genetically distinct second strain of virus (with a median time between positive swabs of 65 days $)^{52}$.

Although the documented cases of reinfection show that this is possible, a major question to address is how likely this is to occur and the level of protection provided by previous infection. A large cohort study compared the incidence of SARS-CoV-2 infection in health-care workers who were either seropositive (indicating recent previous infection) or seronegative for SARS-CoV-2 at enrolment ${ }^{53}$. This study estimated that seropositivity at enrolment reduced the incidence of detected reinfection by almost $89 \%$ over a median follow-up of 139 days. A retrospective study of more than 43,000 participants in a national database in Qatar has estimated a reduction of infection of $95 \%$ in seropositive individuals over a median of 114 days from a seropositive test (preprint data, not yet peer reviewed) ${ }^{54}$. A similar study of more than half a million individuals in Denmark investigated the risk of reinfection in patients who had previously tested positive for SARS-CoV-2 by $\mathrm{PCR}^{55}$. This study estimated a protection of $80.5 \%$ for up to 7 months in patients who had previously tested positive, although a protection of only $47 \%$ was observed in those over 65 years of age. These studies provide evidence for strong protection from reinfection in seropositive individuals during the first few months after infection (at levels similar to the protection seen after vaccination).

In contrast to the relative rarity of documented cases of reinfection in observational studies, a recent vaccine trial in South Africa compared SARS-CoV-2 infection rates in participants who were seropositive and seronegative in their placebo control group. This study found no difference in infection rate between individuals who were seropositive and those who were seronegative (5.2\% compared with $5.3 \%$ ), suggesting that there was no protective effect of previous infection (preprint data, not yet peer reviewed) ${ }^{56}$. However, high levels of transmission of the B.1.351 variant of SARS-CoV-2, which has reduced susceptibility to immune sera from patients infected with ancestral SARS-CoV-2 strains, were occurring at the time of this trial, which may in part explain these findings. A recent seroepidemiological study in the Brazilian city of Manaus has also suggested the potential for reinfection beyond 6-8 months after infection ${ }^{11,12,57}$. Manaus experienced a strong wave of SARS-CoV-2 infection in April and May of 2020, which might have been expected to provide some level of protection or herd immunity in the exposed population. However, despite a low level of transmission from June to December 2020, a resurgence of infection was observed in early 2021 $\left(\mathrm{REFS}^{11,57}\right)$. The role of waning immunity in this apparent wave of reinfection is unclear, as major strain variation was also occurring at the same time $\mathrm{e}^{57,58}$ (BOX 2). However, the contrast between an apparently high level of protection following recent infection ( $<6$ months) and the ongoing spread of infection in a highly seropositive population at later times and/or in the presence of viral antigenic variants raises several important questions.

There is clearly intense interest in understanding the duration and cross-reactivity of immunity elicited by natural infection ${ }^{58,59}$ and its impact on both illness and transmission during reinfection, which has major implications for disease spread and the potential for population-level immunity (BOX 2).

Memory to vaccination versus infection The rapid development and roll-out of vaccines for SARS-CoV-2 has the potential to greatly reduce the morbidity and mortality associated with infection.
A major question is whether vaccineinduced responses can prove to be significantly more effective than natural immunity at controlling infection ${ }^{60}$. Currently licensed vaccines have shown efficacies of up to $95 \%$ in preventing laboratory-confirmed infection over the first few months after vaccination ${ }^{61-63}$, a level of protection that is not significantly different to the $89-95 \%$ protection that has been estimated following natural infection ${ }^{53,54}$. Comparing the magnitude of immune responses to vaccination and infection, the currently licensed vaccines have been shown to produce peak neutralizing antibody responses that range from approximately half to four-times those seen in convalescent patients ${ }^{24,64-67}$ (preprint data, not yet peer reviewed $)^{68}$. Initial comparisons of the durability of both neutralizing and binding antibody responses over the first months after vaccination suggest that the early rate of decay of antibody responses is not significantly different after vaccination or infection $^{24,69}$ (FIG. 1 c). However, if protection requires maintaining antibody levels above a certain threshold, then higher initial levels of response following vaccination or frequent boosting may succeed in keeping antibody levels above this threshold for longer than after natural infection despite similar rates of antibody decay ${ }^{24}$. Interestingly, the vaccination of previously infected individuals drives a rapid and very potent recall of humoral immunity, even after a single vaccine dose $\mathrm{e}^{70-72}$, with serological neutralizing activity generally exceeding levels seen after two vaccine doses in naive participants. The protective potential of heterologous vaccination regimes that might recapitulate this effect warrants further investigation.

These early indications suggest that both infection and vaccination provide significant protection from detectable SARS-CoV-2 infection in the first months after exposure. This contrasts with the seroepidemiological evidence for ongoing spread of infection in a highly exposed population in Manaus, which suggests that there may be susceptibility to reinfection after 6-8 months ${ }^{11,12}$. The relative importance of waning immunity and the circulation of SARS-CoV-2 variants is not yet clear, and it seems likely that both factors may have a role. It is likely that a lower-level or partial immunity reduces the risk of clinical illness and detectable infection to a greater extent than reducing transmission. For example, immunity owing to neutralizing antibodies would be expected to block infection and thereby reduce both illness and transmission 
Box 2 | Antigenic variation and immune recognition

Several emerging viral variants of concern

have been identified that have mutations

in the spike protein, including the B.1.1.7

variant (originating from the UK and which has

eight spike mutations ${ }^{92}$ ), the B.1.351 variant

(originating from South Africa) and the

P.1 variant (originating from Brazil and which

also contains multiple mutations in the

spike protein and is spreading widely) ${ }^{57,93}$.

Concerningly, recent reports suggest that

these variants are partially resistant to

neutralization by monoclonal antibodies

against spike protein, by convalescent plasma

and, notably, by sera from participants who

have received severe acute respiratory

syndrome coronavirus 2 (SARS-CoV-2)

vaccines $^{91,94,95}$.

The emergence and selection of multiple

mutations in the spike protein confirm that the spike is a major target of effective immune responses against SARS-CoV-2. The majority of SARS-CoV-2 vaccines target only the spike protein, although some include the whole inactivated virus and may thus target multiple viral antigens (reviewed in REF ${ }^{96}$ ). However, immune responses following natural infection not only target multiple viral proteins but also induce immune responses that are localized to the tissue sites of initial infection (upper and lower respiratory tracts) (see figure, part a).

Immune responses induced by both infection and vaccination provide good short-term protection from infection with a homologous virus strain. The clinical protection we observe may include contributions from spike-specific and non-spike-specific responses as well as localized responses in the lung (see figure, part b), the relative contribution of which is unknown. Comparing the immune control of SARS-CoV-2 variants following infection or vaccination may enable us to identify the relative importance of tissue-specific, $T$ cell and non-spike responses. For example, if immune control is mediated by neutralizing antibodies to the spike protein, then spike mutations that abrogate antibody recognition alone can abrogate immune protection (whether the
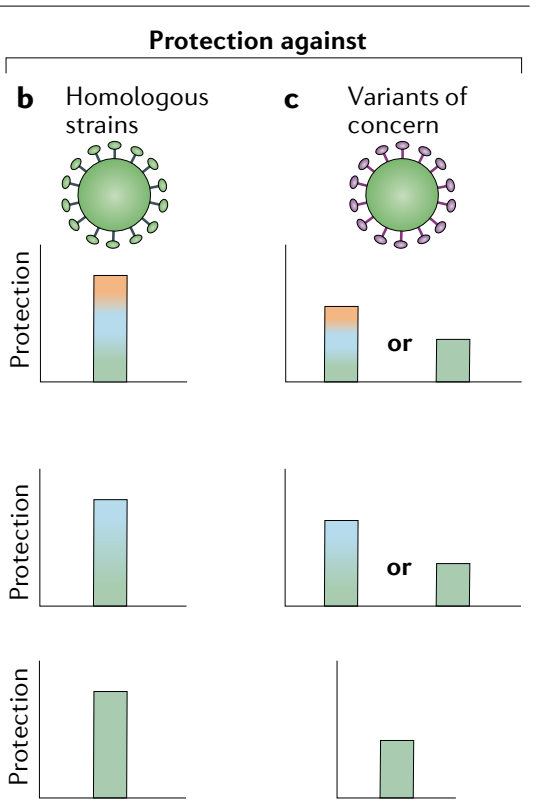

Spike-specific immune responses immune responses

Tissue-specific immune responses

responses are induced by infection or vaccination). However, if responses to non-spike proteins and/or localized responses in the lung are important, then natural infection or vaccination with the whole inactivated virus may maintain better immune control of spike variants, even when neutralization titres towards a variant are low (see figure, part c). Alternatively, if the loss of antibody neutralization abrogates protection even when $T$ cell recognition is maintained, this suggests a primary role for neutralizing antibodies ${ }^{95}$.

Understanding whether immunity from natural infection leads to more durable control of infection or has a greater breadth of recognition of viral variants than vaccination may provide important insights into the effectiveness of different immune mechanisms in controlling SARS-CoV-2 replication.

to a similar extent. However, recall immune responses that allow for early viral replication before they increase to a level able to control peak viral levels might have a greater effect on clinical symptoms than on transmission (BOX 2).

Lessons from hCoVs and influenza virus Predicting the long-term potential for immune control of SARS-CoV-2 is challenging. However, consideration of population-level immunity against endemic hCoVs may provide important insights into the maintenance of long-term immunity against related coronaviruses such as SARS-CoV-2. Endemic hCoVs circulate worldwide and cause widespread infection during childhood ${ }^{73}$. By adulthood, most individuals are likely to have been exposed multiple times, with longitudinal studies and human challenge studies suggesting that individuals become susceptible to reinfection within 12-18 months after a previous hCoV infection ${ }^{74-78}$. Although correlates of protection have not been conclusively identified, studies show that hCoV infection results in a boosting of circulating antibody levels ${ }^{74}$. Interestingly, comparing the rate of decay of antibody responses following $\mathrm{hCoV}$ and SARS-CoV-2 infections suggests that they might have a similar rate of antibody loss (FIG. 1 b). Compared to a large number of studies documenting widespread seroreactivity to all four hCoVs, relatively less is known about $\mathrm{T}$ cell responses to hCoVs. Nonetheless, several studies indicate the presence of widespread $\mathrm{CD} 4^{+}$ $\mathrm{T}$ cell memory to hCoVs among healthy adults ${ }^{32,79-81}$. This presents a picture of an 'endemic equilibrium' between $\mathrm{hCoV}$ infection and human immunity, whereby the rate of decay of immune memory is balanced by regular reinfection and boosting of immune responses, leading to frequent mild infections in the context of short-lived memory ${ }^{82}$. A similar phenomenon of ongoing circulation and frequent reinfection is also observed for influenza virus, although often resulting in more severe clinical outcomes. Importantly, although influenza virus reinfection is often attributed to antigenic variation, human challenge studies show that reinfection with an identical strain is possible within 1 year of initial infection ${ }^{83}$. Combined with evidence for the rapid waning of immune protection after influenza virus vaccination $^{84}$, this suggests that both infection-induced and vaccine-induced immunity to influenza virus are short lived.

Whether SARS-CoV-2 might become less pathogenic in an immune (or semi-immune) population depends on the nature of both the virus and the immune response it elicits. A direct comparison of the relative 


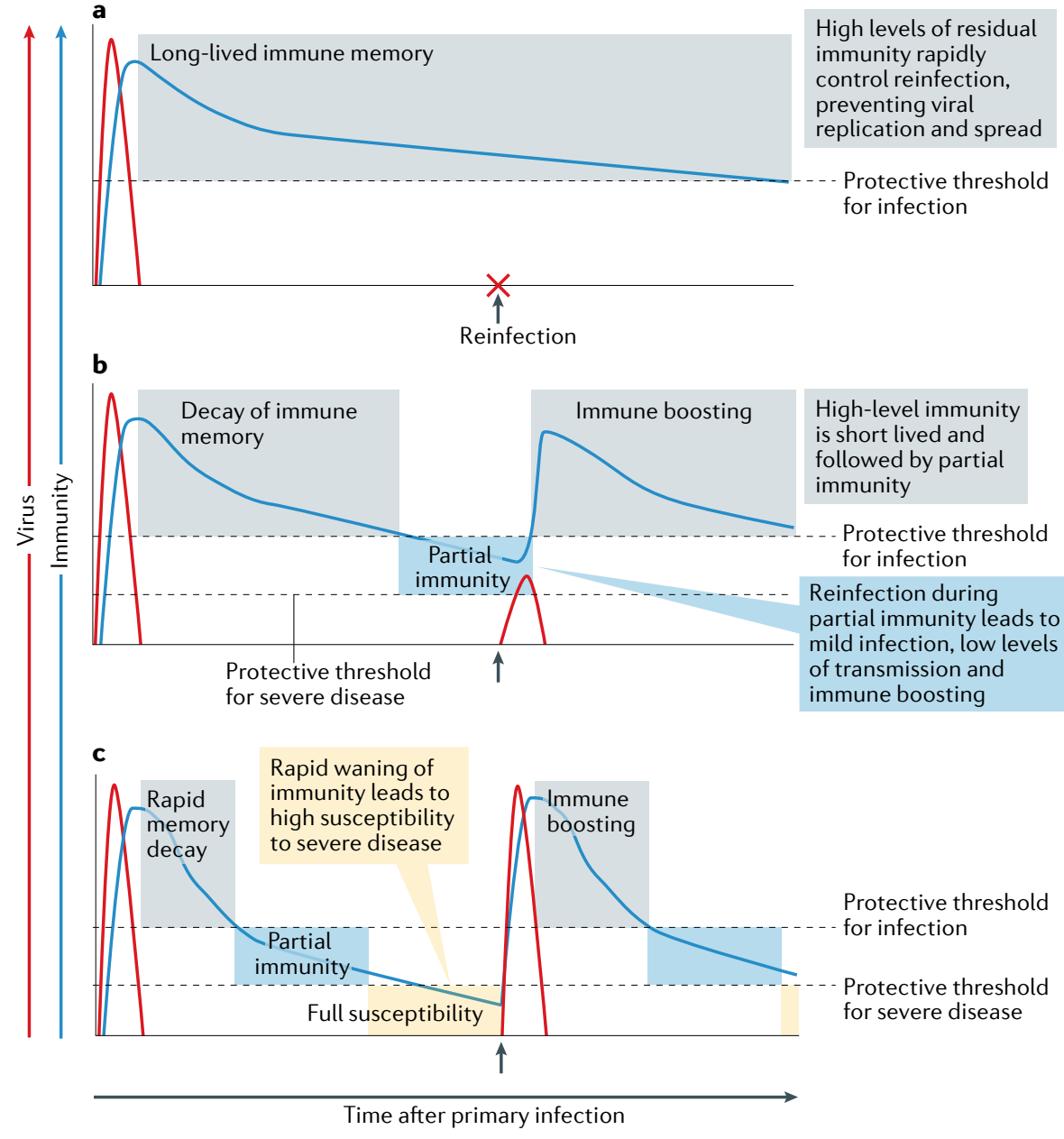

Fig. 3 Alternative trajectories for immunity to SARS-CoV-2. Immune responses, particularly neutralizing antibody responses, to severe acute respiratory syndrome coronavirus 2 (SARS-CoV-2) infection have been observed to decay rapidly in the first few months after infection but the rate of decay seems to slow with time ${ }^{3}$. a In an ideal scenario, immunity remains above a protective threshold for a prolonged period, providing strong immunity from reinfection. $\mathbf{b} \mid$ Immunity may drop below the threshold required to prevent infection but still provide sufficient protection against severe illness. If viral levels after reinfection are high enough to enable transmission, the endemic spread of infection may boost memory responses to maintain immunity at protective levels ${ }^{82}$. $\mathbf{c}$ |Weak immune memory, rapidly decaying immunity or viral antigenic variation may result in the level of immune memory dropping below the threshold required to protect against severe disease, thereby exposing the patient to a similar risk to that seen in primary infection.

virulence and pathogenicity of hCoVs and SARS-CoV-2 is difficult as a defined primary $\mathrm{hCoV}$ infection in naive adults is challenging to identify. However, the natural history of influenza virus pandemics and epidemics may provide a precedent for one potential outcome for SARS-CoV-2. That is, the high pathogenicity of pandemic influenza virus strains during primary infection is reduced to a level equivalent to the pathogenicity of circulating (seasonal and/or endemic) influenza virus strains in the presence of an immune population. The immunological naivety of the host population is thus thought to be a major contributor to the severe illness caused by pandemic influenza ${ }^{85}$.

\section{The future of the pandemic}

Current analyses of the rate of decay of immunity to SARS-CoV-2 and the comparison with immunity to hCoVs suggest that high-level immunity to SARS-CoV-2 infection may last less than a year in many cases but that the duration of protection from severe infection may be more durable ${ }^{24}$. A major question is whether and how vaccination might provide longer lasting or stronger immunity. Potential scenarios for the degree of immune protection conferred by infection and vaccination are shown in FIG. 3. In the most optimistic scenario, vaccination might block virus transmission, leading to control of the pandemic in highly vaccinated populations. However, alternative scenarios are also possible in which vaccine efficacy is either short lived or provides protection from severe disease but does not block transmission ${ }^{74-78}$. Finally, the emergence of antigenic variants of SARS-CoV-2 (BOX 2) raises the possibility of an ongoing 'arms race' between viral evolution and vaccine catch-up (as occurs for annual influenza virus vaccination).

In all but the most optimistic scenarios, it seems that either continued boosting with current vaccines or a next generation of SARS-CoV-2 vaccines may be required, but how then do we optimize viral control? The simplest approach of developing vaccines and/or vaccine schedules that induce stronger neutralizing antibody responses may indeed improve outcomes. For example, although post-infection and post-vaccination responses are currently similar for SARS-CoV-2 $\left(\mathrm{REFS}^{64-68}\right)$, the human papilloma virus vaccine can induce antibody responses that are orders of magnitude larger than those seen in natural infection, and it induces much stronger and more durable immunity than occurs after natural infection ${ }^{86}$. Whether higher vaccine-induced antibody titres to SARS-CoV-2 can be achieved through repeated boosting or will require more immunogenic vaccine formulations is unclear. In addition, expanding the breadth of neutralization against different antigenic variants may be an important requirement of next-generation vaccines.

The concepts of neutralizing antibodies and herd immunity have loomed large in our thinking about SARS-CoV-2 contro $^{87,88}$. Although the neutralization of viral entry into host cells may yet prove to be a dominant pathway to protection, a thorough investigation of different mechanisms of partial immunity and the roles of non-neutralizing antibodies and of $\mathrm{T}$ cell and $\mathrm{B}$ cell memory responses seems prudent. Importantly, vaccines are currently being compared based on their short-term ability to prevent symptomatic infection, with an implicit assumption that this will also reflect their long-term efficacy and ability to control the spread of infection. However, if alternative immune correlates of protection beyond neutralizing antibodies can be established, these may provide additional directions for vaccine optimization and prediction of future protection.

\section{Concluding remarks}

With a growing proportion of the world population having been infected with SARS-CoV-2, there is intense interest in the degree to which this group is protected 
from reinfection in the medium to long term. The waning of neutralizing antibody responses over the first year after infection suggests that reinfections may become more frequent in the coming months and/or years. However, the robust $\mathrm{B}$ cell and $\mathrm{T}$ cell memory responses induced by primary infection suggest that reinfection severity, and potentially transmission, may be mitigated over the longer term. The potential for higher levels of neutralizing antibodies to be induced by vaccination suggests that reinfection could be further reduced by vaccinating individuals who have been previously infected. Nevertheless, the durability of immunity and the potential for antigenic variation of the virus remain major challenges going forwards. A better understanding of the mechanisms of immunity against reinfection is urgently needed to help achieve long-term immune control of the SARS-CoV-2 pandemic.

\section{Deborah Cromer 1,3, Jennifer A. Juno iD 2,3, David Khoury iD ', Arnold Reynaldi iD',} Adam K. Wheatley (D) 2, Stephen J. Kent ${ }^{2,3}$ 凶 and Miles P. Davenport iD ${ }^{1,3 \otimes}$

${ }^{1}$ Kirby Institute, University of New South Wales, Sydney, Australia.

2Department of Microbiology and Immunology, University of Melbourne at the Peter Doherty Institute for Infection and Immunity, Melbourne, Australia.

${ }^{3}$ These authors contributed equally: Deborah Cromer Jennifer A. Juno, Stephen J. Kent, Miles P. Davenport.

凶e-mail:skent@unimelb.edu.au;m.davenport@ unsw.edu.au

https://doi.org/10.1038/s41577-021-00550-x

Published online 29 Apr 2021

Sette, A. $\&$ Crotty, S. Adaptive immunity to SARS-CoV-2 and COVID-19. Cell 184, 861-880 (2021).

2. Gaebler, C. et al. Evolution of antibody immunity to SARS-CoV-2. Nature 591, 639-644 (2021).

3. Wheatley, A. K. et al. Evolution of immune responses to SARS-CoV-2 in mild-moderate COVID-19. Nat. Commun. 12, 1162 (2021).

4. Robbiani, D. F. et al. Convergent antibody responses to SARS-CoV- 2 in convalescent individuals. Nature 584, 437-442 (2020).

5. Muecksch, F. et al. Longitudinal serological analysis and neutralizing antibody levels in coronavirus disease 2019 convalescent patients. J. Infect. Dis. 223 389-398 (2021)

6. Seow, J. et al. Longitudinal observation and decline of neutralizing antibody responses in the three months following SARS-CoV-2 infection in humans. Nat Microbiol. 5, 1598-1607 (2020).

7. Wajnberg, A. et al. Robust neutralizing antibodies to SARS-CoV-2 infection persist for months. Science 370 1227-1230 (2020)

8. Dan, J. M. et al. Immunological memory to SARS-CoV-2 assessed for up to 8 months after infection. Science 371, eabf4063 (2021)

9. Rodda, L. B. et al. Functional SARS-CoV-2-specific immune memory persists after mild COVID-19. Cell 184, 169-183.e117 (2021).

10. Zuo, J. et al. Robust SARS-CoV-2-specific T cell immunity is maintained at 6 months following primary infection. Nat. Immunol. https://doi.org/10.1038/ s41590-021-00902-8 (2021).

11. Buss, L. F. et al. Three-quarters attack rate of SARS-CoV-2 in the Brazilian Amazon during a largely unmitigated epidemic. Science 371, 288-292 (2021)

12. Sridhar, D. \& Gurdasani, D. Herd immunity by infection is not an option. Science 371, 230-231 (2021).
13. Röltgen, K. et al. Defining the features and duration of antibody responses to SARS-CoV-2 infection associated with disease severity and outcome. Sci. Immunol. 5, eabe0240 (2020).

14. Ibarrondo, F. J. et al. Rapid decay of anti-SARS-CoV-2 antibodies in persons with mild Covid-19. N. Engl. J. Med. 383, 1085-1087 (2020).

15. Crawford, K. H. D. et al. Dynamics of neutralizing antibody titers in the months after SARS-CoV-2 infection. J. Infect. Dis. 223, 197-205 (2020)

16. Wu, J. et al. SARS-CoV-2 infection induces sustained humoral immune responses in convalescent patients following symptomatic COVID-19. Nat. Commun. 12, 1813 (2021)

17. Beaudoin-Bussières, G. et al. Decline of humoral responses against SARS-CoV-2 spike in convalescent individuals. mBio 11, e02590-20 (2020).

18. Yao, X.-Y. et al. Neutralizing and binding antibody kinetics of COVID-19 patients during hospital and convalescent phases. Preprint at medRxiv https:/ doi.org/10.1101/2020.07.18.20156810 (2020).

19. Sokal, A. et al. Maturation and persistence of the anti-SARS-CoV-2 memory B cell response. Cell 184 1201-1213.e14 (2021)

20. Ellebedy, A. et al. SARS-CoV-2 infection induces longlived bone marrow plasma cells in humans. Preprint at Res. Sq. https://doi.org/10.21203/rs.3.rs-132821/v1 (2020).

21. Hammarlund, E. et al. Plasma cell survival in the absence of B cell memory. Nat. Commun. 8, 178 (2017).

22. Antia, A. et al. Heterogeneity and longevity of antibody memory to viruses and vaccines. PLOS Biol. 16, e2006601 (2018).

23. Amanna, I. J., Carlson, N. E. \& Slifka, M. K. Duration of humoral immunity to common viral and vaccine antigens. N. Engl. J. Med. 357, 1903-1915 (2007).

24. Khoury, D. S. et al. What level of neutralising antibody protects from COVID-19? Preprint at medRxiv https:/ doi.org/10.1101/2021.03.09.21252641 (2021).

25. Schafer, A. et al. Antibody potency, effector function, and combinations in protection and therapy for SARS-CoV-2 infection in vivo. J. Exp. Med. 218 e20201993 (2021)

26. Lee, W. S. et al. Decay of Fc-dependent antibody functions after mild to moderate COVID-19. Preprint at medRxiv https://doi.org/10.1101/ 2020.12.13.20248143 (2020).

27. Winkler, E. S. et al. Human neutralizing antibodies against SARS-CoV-2 require intact Fc effector functions for optimal therapeutic protection. Cell 184, 1804-1820.e16 (2021).

28. Hartley, G. E. et al. Rapid generation of durable B cell memory to SARS-CoV-2 spike and nucleocapsid proteins in COVID-19 and convalescence. Sci. Immunol. 5, eabf8891 (2020).

29. Jiang, X. L. et al. Lasting antibody and $T$ cell responses to SARS-CoV-2 in COVID-19 patients three months after infection. Nat. Commun. 12, 897 (2021).

30. Bilich, T. et al. T cell and antibody kinetics delineate SARS-CoV-2 peptides mediating long-term immune responses in COVID-19 convalescent individuals. Sci. Trans/ Med. https://doi.org/10.1126/scitranslmed. abf7517 (2021).

31. Rydyznski Moderbacher, C. et al. Antigen-specific adaptive immunity to SARS-CoV-2 in acute COVID-19 and associations with age and disease severity. Cell 183, 996-1012.e19 (2020).

32. Juno, J. A. et al. Humoral and circulating follicular helper $\mathrm{T}$ cell responses in recovered patients with COVID-19. Nat. Med. 26, 1428-1434 (2020).

33. Hufford, M. M., Kim, T. S., Sun, J. \& Braciale, T. J. The effector $\mathrm{T}$ cell response to influenza infection. Curr. Top. Microbiol. Immunol. 386, 423-455 (2015)

34. Pizzolla, A. et al. Resident memory $\mathrm{CD}^{+} \mathrm{T}$ cells in the upper respiratory tract prevent pulmonary influenza virus infection. Sci. Immunol. 2, eaam6970 (2017).

35. Allie S. R et al. The establishment of resident memory $\mathrm{B}$ cells in the lung requires local antigen encounter. Nat. Immunol. 20, 97-108 (2019).

36. Weinreich, D. M. et al. REGN-COV2, a neutralizing antibody cocktail, in outpatients with Covid-19. N. Engl. J. Med. 384, 238-251 (2020).

37. Chen, P. et al. SARS-CoV-2 neutralizing antibody LY-CoV555 in outpatients with Covid-19. N. Engl. J. Med. 384, 229-237 (2020)

38. Libster, R. et al. Early high-titer plasma therapy to prevent severe covid-19 in older adults. N. Engl. J. Med. 384, 610-618 (2021).

39. Joyner, M. J. et al. Convalescent plasma antibody levels and the risk of death from Covid-19. N. Engl. J. Med. 384, 1015-1027 (2021).
40. Group, A.-T. L.-C. S. et al. A neutralizing monoclonal antibody for hospitalized patients with Covid-19. N. Engl. J. Med. 384, 905-914 (2021).

41. Cohen, M. S. Monoclonal antibodies to disrupt progression of early covid-19 infection. N. Engl. J. Med. 384, 289-291 (2021).

42. Wolf, A. J. et al. Initiation of the adaptive immune response to Mycobacterium tuberculosis depends on antigen production in the local lymph node, not the lungs. J. Exp. Med. 205, 105-115 (2008).

43. Davenport, M. P., Ribeiro, R. M. \& Perelson, A. S Kinetics of virus-specific CD8 ${ }^{+} \mathrm{T}$ cells and the contro of human immunodeficiency virus infection. J. Virol. 78, 10096-10103 (2004).

44. Reynolds, M. R. et al. CD8 ${ }^{+}$T-lymphocyte response to major immunodominant epitopes after vaginal exposure to simian immunodeficiency virus: too late and too little. J. Virol. 79, 9228-9235 (2005).

45. Davenport, M. P., Belz, G. T. \& Ribeiro, R. M. The race between infection and immunity: how do pathogens set the pace? Trends Immunol. 30, 61-66 (2009).

46. Deng, W. et al. Primary exposure to SARS-CoV-2 protects against reinfection in rhesus macaques. Science 369, 818-823 (2020)

47. Chandrashekar, A. et al. SARS-CoV-2 infection protects against rechallenge in rhesus macaques. Science 369 , 812-817 (2020)

48. McMahan, K. et al. Correlates of protection against SARS-CoV-2 in rhesus macaques. Nature $\mathbf{5 9 0}$, 630-634 (2021).

49. Khoury, D. S et al. Measuring immunity to SARS-CoV-2 infection: comparing assays and animal models. Nat. Rev. Immunol. 20, 727-738 (2020).

50. Larson, D. et al. A case of early re-infection with SARS-CoV-2. Clin. Infect. Dis. https://doi.org/10.1093/ cid/ciaa1436 (2020).

51. To, K. K. et al. COVID-19 re-infection by a phylogenetically distinct SARS-coronavirus- 2 strain confirmed by whole genome sequencing. Clin. Infect. Dis. https://doi.org/10.1093/cid/ciaa1275 (2020).

52. Abu-Raddad, L. J. et al. Assessment of the risk of SARS-CoV-2 reinfection in an intense re-exposure setting. Clin. Infect. Dis. https://doi.org/10.1093/ $\mathrm{cid} / \mathrm{ciaa} 1846$ (2020).

53. Lumley, S. F. et al. Antibody status and incidence of SARS-CoV-2 infection in health care workers. N. Engl. J. Med. 384, 533-540 (2021).

54. Abu-Raddad, L. J. et al. SARS-CoV-2 reinfection in a cohort of 43,000 antibody-positive individuals followed for up to 35 weeks. Preprint at medRxiv https://doi.org/10.1101/2021.01.15.21249731 (2021).

55. Hansen, C. H., Michlmayr, D., Gubbels, S. M. Mølbak, K. \& Ethelberg, S. Assessment of protection against reinfection with SARS-CoV- 2 among 4 million PCR-tested individuals in Denmark in 2020: a population-level observational study. Lancet 397 1204-1212 (2021).

56. Shinde, V. et al. Preliminary efficacy of the NVXCoV2373 Covid-19 vaccine against the B.1.351 variant. Preprint at medRxiv https://doi.org/10.1101/ 2021.02.25.21252477 (2021).

57. Sabino, E. C. et al. Resurgence of COVID-19 in Manaus, Brazil, despite high seroprevalence. Lancet 397, 452-455 (2021)

58. Chen, R. E. et al. Resistance of SARS-CoV-2 variants to neutralization by monoclonal and serum-derived polyclonal antibodies. Nat. Med. https://doi.org/ 10.1038/s41591-021-01294-w (2021)

59. Liu, Y. et al. Neutralizing activity of BNT162b2elicited serum - preliminary report. N. Engl. J. Med. https://doi.org/10.1056/NEJMc2102017 (2021).

60. Burton, D. R. \& Topol, E. J. Toward superhuman SARS-CoV-2 immunity? Nat. Med. 27, 5-6 (2021)

61. Polack, F. P. et al. Safety and efficacy of the BNT162b2 mRNA Covid-19 vaccine. N. Engl. J. Med. 383 , 2603-2615 (2020).

62. Baden, L. R. et al. Efficacy and safety of the mRNA 1273 SARS-CoV-2 vaccine. N. Engl. J. Med. 384 403-416 (2020).

63. Sadoff, J. et al. Interim results of a phase 1-2a trial of Ad26. COV2. S covid-19 vaccine. N. Engl. J. Med. https://doi.org/10.1056/NEJMoa2034201 (2021).

64. Zhang, Y. et al. Safety, tolerability, and immunogenicity of an inactivated SARS-CoV-2 vaccine in healthy adults aged 18-59 years: a randomised, double-blind, placebo-controlled, phase $1 / 2$ clinical trial. Lancet Infect. Dis. 21, 181-192 (2021).

65. Folegatti, P. M. et al. Safety and immunogenicity of the ChAdOx $1 \mathrm{nCoV}-19$ vaccine against SARS-CoV-2: a preliminary report of a phase $1 / 2$, single-blind, 
randomised controlled trial. Lancet 396, 467-478 (2020).

66. Walsh, E. E. et al. Safety and immunogenicity of two RNA-based covid-19 vaccine candidates. N. Engl. J. Med. 383, 2439-2450 (2020).

67. Jackson, L. A. et al. An mRNA vaccine against SARS-CoV-2 - preliminary report. N. Engl. J. Med. 383, 1920-1931 (2020)

68. Sahin, U. et al. BNT162b2 induces SARS-CoV-2neutralising antibodies and T cells in humans. Preprint at medRxiv https://doi.org/10.1101/ 2020.12.09.20245175 (2020).

69. Widge, A. T. et al. Durability of responses after SARS-CoV-2 mRNA-1273 vaccination. N. Engl. J. Med. 384, 80-82 (2021).

70. Krammer, F. et al. Antibody responses in seropositive persons after a single dose of SARS-CoV-2 mRNA vaccine. N. Engl. J. Med. https://doi.org/10.1056/ NEJMc2101667 (2021)

71. Prendecki, M. et al. Effect of previous SARS-CoV-2 infection on humoral and T-cell responses to singledose BNT162b2 vaccine. Lancet 397, 1178-1181 (2021).

72. Manisty, C. et al. Antibody response to first BNT162b2 dose in previously SARS-CoV-2-infected individuals. Lancet 397, 1057-1058 (2021).

73. Zhou, W., Wang, W., Wang, H., Lu, R. \& Tan, W. First infection by all four non-severe acute respiratory syndrome human coronaviruses takes place during childhood. BMC Infect. Dis. 13, 433-433 (2013).

74. Edridge, A. W. D. et al. Seasonal coronavirus protective immunity is short-lasting. Nat. Med. 26, 1691-1693 (2020)

75. Galanti, M. \& Shaman, J. Direct observation of repeated infections with endemic coronaviruses. J. Infect. Dis. 223, 409-415 (2020).

76. Callow, K. A., Parry, H. F., Sergeant, M. \& Tyrrell, D. A The time course of the immune response to experimental coronavirus infection of man. Epidemiol. Infect. 105, 435-446 (1990).

77. Callow, K. A. Effect of specific humoral immunity and some non-specific factors on resistance of volunteers to respiratory coronavirus infection. J. Hyg. 95 173-189 (1985)

78. Lepiller, Q. et al. High incidence but low burden of coronaviruses and preferential associations between respiratory viruses. J. Clin. Microbiol. 51, 3039-3046 (2013)

79. Bacher, P. et al. Low-avidity $\mathrm{CD}^{+} \mathrm{T}$ cell responses to SARS-COV-2 in unexposed individuals and humans with severe COVID-19. Immunity 53, 1258-1271.e5 (2020).

80. Woldemeskel, B. A. et al. Healthy donor T cell responses to common cold coronaviruses and SARS-CoV-2. J. Clin. Invest. 130, 6631-6638 (2020).

81. Mok, C. K. P. et al. T-cell responses to MERS coronavirus infection in people with occupational exposure to dromedary camels in Nigeria: an observational cohort study. Lancet Infect. Dis. 21, 385-395 (2020).

82. Lavine, J. S., Bjornstad, O. N. \& Antia, R. Immunological characteristics govern the transition of COVID-19 to endemicity. Science 371, 741-745 (2021).

83. Memoli, M. J. et al. Influenza a reinfection in sequential human challenge: implications for protective immunity and "Universal" vaccine development. Clin. Infect. Dis. 70, 748-753 (2020).

84. Ferdinands, J. M. et al. Intraseason waning of influenza vaccine protection: evidence from the US Influenza Vaccine Effectiveness Network, 2011-12 through 2014-15. Clin. Infect. Dis. 64, 544-550 (2017).

85. Taubenberger, J. K. \& Morens, D. M. 1918 Influenza: the mother of all pandemics. Emerg. Infect. Dis. 12, 15-22 (2006)

86. Petaja, $T$ et al. Long-term persistence of systemic and mucosal immune response to HPV-16/18 ASO4 adjuvanted vaccine in preteen/adolescent girls and young women. Int. J. Cancer 129, 2147-2157 (2011).

87. Randolph, H. E. \& Barreiro, L. B. Herd immunity: understanding COVID-19. Immunity 52, 737-741 (2020).

88. Anderson, R. M., Vegvari, C., Truscott, J. ¿ Collyer, B. S. Challenges in creating herd immunity to SARS-CoV-2 infection by mass vaccination. Lance 396, 1614-1616 (2020)

89. Liu, W. et al. Two-year prospective study of the humoral immune response of patients with severe acute respiratory syndrome. J. Infect. Dis. 193 792-795 (2006)

90. Tang, F. et al. Lack of peripheral memory B cell responses in recovered patients with severe acute respiratory syndrome: a six-year follow-up study. J. Immunol. 186, 7264-7268 (2011).

91. Wang, Z. et al. mRNA vaccine-elicited antibodies to SARS-CoV-2 and circulating variants. Nature https:/ doi.org/10.1038/s41586-021-03324-6 (2021).

92. Davies, N. G. et al. Estimated transmissibility and impact of SARS-CoV-2 lineage B.1.1.7 in England.
Science https://doi.org/10.1126/science.abg3055 (2021).

93. Tegally, H. et al. Sixteen novel lineages of SARS-CoV-2 in South Africa. Nat. Med. 27, 440-446 (2021).

94. Wibmer, C. K. et al. SARS-CoV-2 501Y.V2 escapes neutralization by South African COVID-19 donor plasma. Nat. Med. https://doi.org/10.1038/ s41591-021-01285-x (2021).

95. Madhi, S. A. et al. Efficacy of the ChAdOx $1 \mathrm{nCoV}-19$ covid-19 vaccine against the B.1.351 variant. N. Engl. J. Med. https://doi.org/10.1056/NEJMoa2102214 (2021).

96. Krammer, F. SARS-CoV-2 vaccines in development. Nature 586, 516-527 (2020).

\section{Acknowledgements}

This study was supported by an Australian government Medical Research Future Fund award GNT2002073 (S.J.K., M.P.D. and A.K.W.), MRF2005544 (S.J.K., A.K.W., J.A.J. and M.P.D.), MRF2005760 (M.P.D.), the ARC Centre of Excellence in Convergent Bio-Nano Science and Technology (S.J.K.), an NHMRC program grant APP11 49990 (S.J.K. and M.P.D.), NHMRC project grant GNT1162760 (A.K.W.), an NHMRC-EU collaborative award APP 1115828 (S.J.K. and M.P.D.), and the European Union Horizon 2020 Research and Innovation Programme under grant agreement 681137 (S.J.K.). D.C., J.A.J., D.K., A.K.W., S.J.K. and M.P.D. are funded by NHMRC Fellowship/Investigator grants.

\section{Author contributions}

All authors researched data for the article, contributed substantially to discussion of the content, wrote the article, and reviewed and/or edited the manuscript before submission.

\section{Competing interests}

The authors declare no competing interests.

\section{Peer review information}

Nature Reviews Immunology thanks R. Cox and the other, anonymous, reviewer(s) for their contribution to the peer review of this work.

Publisher's note

Springer Nature remains neutral with regard to jurisdictional claims in published maps and institutional affiliations.

\section{Supplementary information}

The online version contains supplementary material available at https://doi.org/10.1038/s41577-021-00550-x.

(c) Springer Nature Limited 2021 